\title{
CNN Models of Receptive Field Dynamics of the Central Visual System Neurons.
}

\author{
László Orzó ${ }^{1}$, Károly Lászlón ${ }^{2}$ László Négyessy ${ }^{l}$, \\ József Hámori ${ }^{1}$, Tamás Roska ${ }^{2}$ \\ ${ }^{1}$ Laboratory of Neurobiology, Department of Anatomy, Semmelweis Medical School, \\ Tûzoltó u. 58, H-1450, Budapest, HUNGARY \\ ${ }^{2}$ Neuromorphic Information Technology, Graduate Center Úri u. 49, H-1014, Budapest, \\ HUNGARY
}

\begin{abstract}
This paper deals with the biological aspects of the receptive field (RF) concept and its possible Cellular Neural Network (CNN) modeling. Three kinds of receptive field definitions are discussed: the experimentally measured $R F$, the mathematical model of the RF and its anatomical background. Recently, new RF-mapping techniques have revealed that neurons in the visual pathway exhibit striking $R F$ dynamics [3], which implicates that for adequate characterization, the $R F$ profile has to be examined in the space-time domain. Starting from these findings in the present study the neurons' static RF definition is purified, and some experimental results of De Angelis et al. [3] is modeled by the CNN [1, 2]. Our CNN model indicates that the spatio-temporal RF dynamics can be generated by time invariant synaptic strength values.
\end{abstract}

\section{Introduction}

The goal of this paper is to provide a RF definition acceptable by neuroscientists but simultaneously giving the possibility of its mathematical modeling. For this purpose the $\mathrm{CNN}$, which is a reliable paradigm for modeling the major components of the visual system [4, 8, 10, 11], is used. Some prospective practical applications will be also discussed..

The RF is a fundamental concept in neuroscience. It is frequently used in the study of neurons, analyzing the transformation between different characteristics of the stimuli and the elicited neuronal activity. The RF expresses the relationship between the stimuli and the evoked neural responses. The measuring method and its theoretical background are defining the measured or phenomenological $R F$. Nevertheless, the receptive field organization has to be also examined from the anatomical point of view, particularly considering the nervous systems' modular organization. If we want to simulate the measured data, we have to choose a model. The CNN seems to be applicable as a generic model of different neuronal systems. Major components of the visual system (retina, LGN, primary visual cortex) have already been modeled by CNN [4, 8, 10, 11]. Utilizing a given mathematical definition the CNN model of the RF can also be obtained. With this model, due to the CNN's biological applicability, we can achieve deeper insight into the fundamental construction of connectivity and physiology of the otherwise very complicated multi-level visual system. It is known that the RFs of the visually responsive neurons exhibit well-defined spatial structure. However, using new techniques of measurements it became evident recently, that the visually responsive neurons show dynamic, spatio-temporal RF properties. We shall show in this paper that the same dynamics as the measured ones [3] can be generated by time invariant CNN templates. The mathematical definition of the RF is simplified in this paper; instead more realistic, nonlinear models will be also required.

\section{Receptive Field Definitions}

\subsection{Anatomical Backgrounds}

The concept of RF of the visual system neurons was based mostly on the anatomical organization of the visual system [5]. In fact, the results of RF measurements and the anatomical data are occasionally in good correlation. However, the RF can not be adequately defined solely on the basis of interconnection patterns in such a highly structured system. In addition, anatomically defined connection patterns can dynamically change in various states of light adaptation [12], and can lead to the so called "dynamic change" of the receptive field structure. It is important to mention that for RF measurements adequate visual stimuli have to be used. Using artificial stimulation and measuring the evoked responses from the corresponding layer of the visual pathway (e.g., 
stimulating and recording within the primary visual cortex) gives information about the physiological properties of the underlying network, but do not determine any kind of receptive field. The receptive field is defined instead by the interaction of the ascending inputs and the horizontal connections within the neural network.

\subsection{Measured Visual Receptive Field}

Definition: The receptive field definition is based on the method of the measurement . The following algorithm is used for RF measurements in the visual system: select a single neuron in any part of the visual pathway, e.g., either in the retina, in the LGN, or in the primary visual cortex. The output (response for the specific stimulus) of this cell is measured. The stimulus during the experiment is a bar or a spot of light projected to the retina. Scan the retina with the stimulus and define the area that has any measurable (above the noise level) influence on the measured neuron response. The scanned points indicate the excitatory or inhibitory parts of the receptive field of the measured cell. The profile of these points describes the measured receptive field.

The RF is inherently a dynamic structure, and the response is a spatio-temporal function of the stimuli. To determine the neurons' dynamic receptive field several types of stimulation paradigms are used (For review see [3]).

It is important to remark, that the duration of the (bar or spot of light) stimulus should be short and there should be enough time between the consecutive stimulation. If the flashed stimuli do not satisfy these conditions, it can not reveal the intrinsic temporal dynamics of the receptive field. In this case a step response will be measured, rather than an impulse response.

\subsection{Mathematical Model of the Receptive Field by Cellular Neural Networks}

With the aid of measurements the neurons' responses to a given stimulus can be determined. If the neuron's operation is nonlinear, the receptive field can be characterized by the output: $f(k, 1, t)$. On the other hand, if the neurons' receptive field is a linear function, both space and time, then we can fully describe the systems' output by a transfer function. Experimental data show that this is the situation in the case of the retinal ganglion cells, the LGN neurons, and the cortical simple cells' in a circumscribed range of the visual stimuli. In these cases we can define the mathematical model of a receptive field as impulse response function (transfer function) of the neurons. It is important to note, that the neuron's transfer function is not the property of an individual cell but of the whole underlying network.

Although, the next definition seems to be simplistic, since it is linear (and we know that in reality it is not the case), it seems to reflect certain basic properties of the RF.

Definition: A single neuron can be considered as a SPATIO-TEMPORAL operator.

Assuming that the neuron's function is linear, the impulse-response can be computed. Such a response (denoted with $h(i, j, t)$ ) is defined as the output due to an input of the type:

$$
u(k, l, t)=\delta_{k, i} \cdot \delta_{l, j} \cdot \delta(t)
$$

where $\delta_{\mathrm{k}, \mathrm{i}} \delta_{\mathrm{l}, \mathrm{j}}$ are the Kronecker delta function and $\delta(\mathrm{t})$ is the Dirac-delta function, which represents a bright or dark impulse. The neuron's response to an arbitrary stimulus can be expressed by

$$
f(k, l, t)={ }_{0 k^{\prime}, l^{\prime}}^{t} u\left(k-k^{\prime}, l-l^{\prime}, t-t^{\prime}\right) \cdot h\left(k^{\prime}, l^{\prime}, t^{\prime}\right) \cdot d t^{\prime} .
$$

The receptive field may be defined by the area of visual space satisfying the following property:

$$
\exists t: h(k, l, t) \neq 0
$$

With the use of the concept of the transfer function, both static and dynamic receptive field definitions can be formulated.

For the experimental measurement of the neurons' transfer function there are different methods that can be used. The first method ('response plane' techniques) uses Dirac-delta (or practically impulses) as input and the measurement of the network output for such stimulus. However, in the case of neurons it is more convenient to record only from one cell at one time and mapping the RF's spatial and temporal properties by changing the position of the stimulus successively [6]. This type of method is relatively slow, because we need to collect the response data sequentially. Another method uses continuous recordings from a cell utilizing pseudo-random spatio-temporal stimuli. It defines the transfer function from the correlation between the evoked neuronal spike train and the stimulus sequence (cross- or reverse-correlation), i.e. it uses white-noise analysis [7].

There could be other aspects of the receptive field concept which incorporates other types of effects of the stimulus on the neuron's response. One of these could be the fine temporal structure of the response to the 
elicited sequence of action potential. By synchronizing the emitted action potentials of different neurons more information could be acquired [10] about the visual stimulus.

In this paper, our purpose is to show, how the same output can be generated in different models.

\section{Simulation and results}

Using the $\mathrm{CNN}$ as a modeling frame [9] we can make a phenomenological model of the dynamics and the spatial properties of the retinal ganglion cells', the LGN neurons' and the cortical simple cells' transfer functions. Our models may not be physiologically "faithful", but might provide new, qualitative information.

\subsection{Dynamics Generation}

The model generates first the temporal characteristics of the output (dynamics). For the generation of it we use four layers of neurons: The first layer (Fig 1.) slows down the input by an appropriate feedback template :

$$
A_{11}=[0.7] \quad B=[1]
$$

The second layer (Fig 1.) generates the faster ('excitatory') part of the response:

$$
A_{12}=[0.5] \quad A_{22}=[0.3]
$$

And the third layer (Fig 1.) generates the slower ('inhibitory') part of the response:

$$
A_{13}=[-0.15] \quad A_{33}=[0.9]
$$

The sum of the second and the third layer can reproduce the bimodal response of the neurons for a 'Dirac delta', impulse stimulus (Fig 1.).

$$
A_{24}=[1] \quad A_{34}=[0.7]
$$

In the case of 'Off' cells only the sign of these template values has to be changed to its reverse.

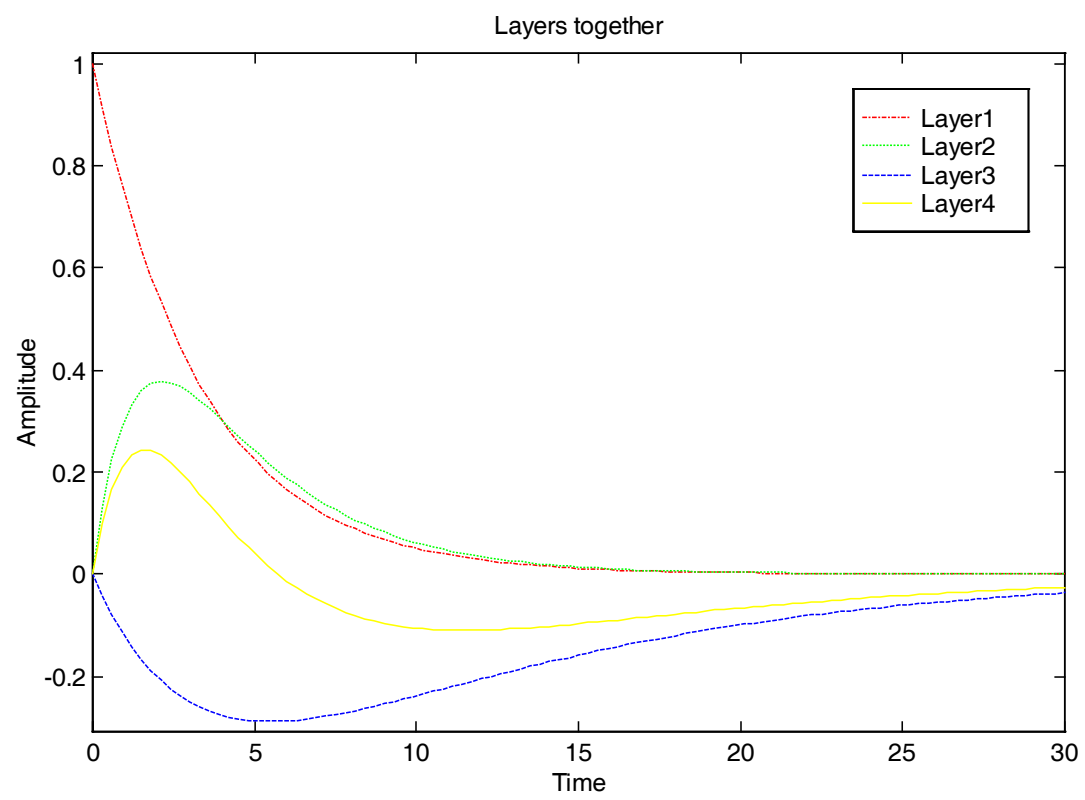

Figure 1. The output of the layers plotted together

\subsection{Spatial Convergence}

A suitable convergence of responses can generate either the center or the surround of the receptive field: 


$$
A_{45(\text { (enter) }}=\left|\begin{array}{lll}
2 & 2 & 2 \\
2 & 3 & 2 \\
2 & 2 & 2
\end{array}\right| \quad A_{45 \text { (SURROUND) }}=\left|\begin{array}{ccccccc}
-1 & -1 & -1 & -1 & -1 & -1 & -1 \\
-1 & -1.2 & -1.2 & -1.2 & -1.2 & -1.2 & -1 \\
-1 & -1.2 & -1.2 & -1.2 & -1.2 & -1.2 & -1 \\
-1 & -1.2 & -1.2 & -1.2 & -1.2 & -1.2 & -1 \\
-1 & -1 & -1 & -1 & -1 & -1 & -1
\end{array}\right|
$$

This CNN model can reproduce the retinal ganglion (or the same kind of thalamic) cells' transfer function. On Fig. 2 we can compare the measured and modeled transfer functions, as expressed in cortical simple cells'. All the following measurement results were taken from [3].
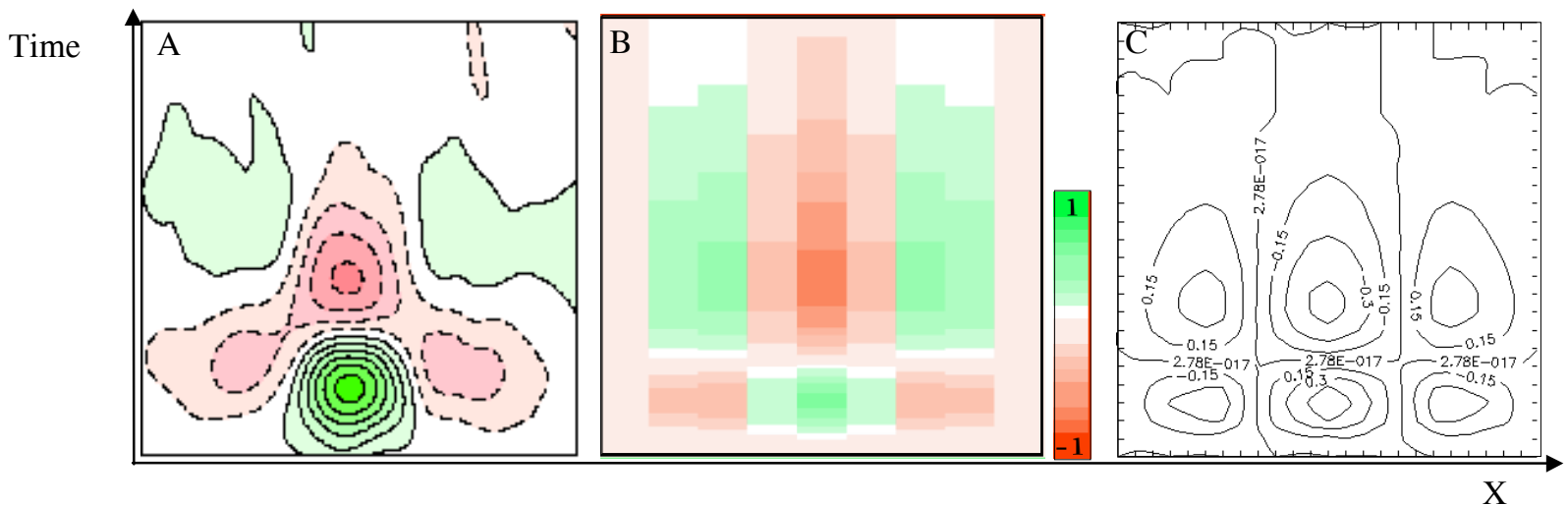

Figure 2. The measured $(A)$ and the $C N N$ modeled $(B, C)$ cortical simple cell's receptive fields. Here and on the following figures $B$ is the false colored and $C$ the topographic representation of the CNN model results. The amount of green color indicates excitation and the red shows inhibition.

For the modeling of the lagged LGN cells' response we have to change the temporal characteristics of the previous model. The lagged cells' delayed response seems to be an inherent characteristic of these cells, not depending of its' retinal input. We can use a simple template to obtain this type of property:

$$
\mathrm{A}_{56}=[0.7] \quad \mathrm{A}_{66}=[0.4]
$$

The modeled lagged cell's transfer function compared with the measured response can be seen on Fig. 3 .

Time
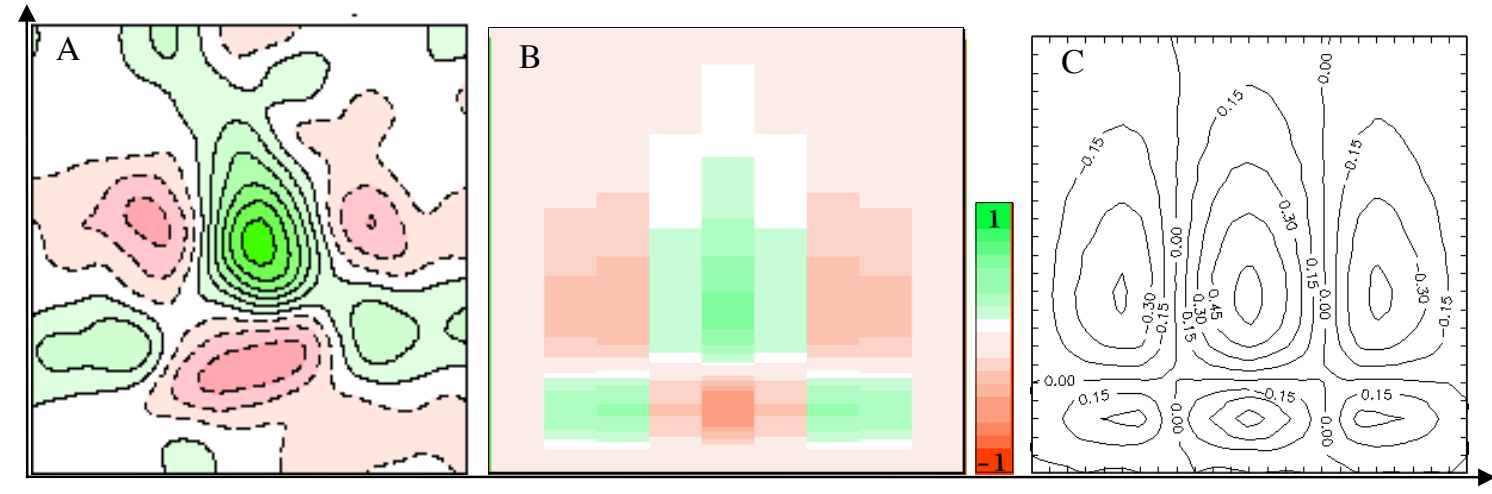

$\mathrm{X}$

Figure 3. We can compare the measured (A) and the modeled $(B, C)$ lagged cells' receptive fields. The model was made by an appropriate "slow down" of a simple cell response (see Fig. 2.) by feedback. The time-course of the response is much slower (not shown), than in the case of simple cells.

From these two types of transfer functions we can build the models of the cortical simple cells' transfer functions. The first class of the cortical simple cells, although they have orientation selective properties (there is small change in y direction), in the $\mathrm{x}$-t map of the RF are similar to the LGN X cells (Fig. 2).

The second class of the cortical simple cells are time separable, and has spatially bimodal center-surround organization in its transfer function. To model this type of receptive field structure we sum two simple transfer functions (On and Off ones) with a small spatial shift. In the temporal domain there is no change in the model. The next templates give the appropriate receptive field: 


$$
A_{57}=\left|\begin{array}{lll}
0 & 0 & 0 \\
0 & 0 & 1 \\
0 & 0 & 0
\end{array}\right| \quad A_{57}=\left|\begin{array}{ccc}
0 & 0 & 0 \\
0 & -1 & 0 \\
0 & 0 & 0
\end{array}\right|
$$

The resulting transfer function can be seen on Fig. 4 .

Time
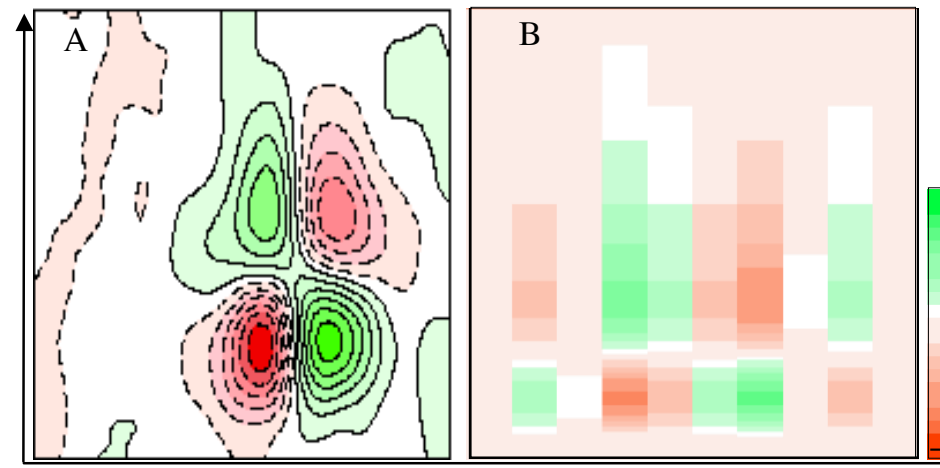

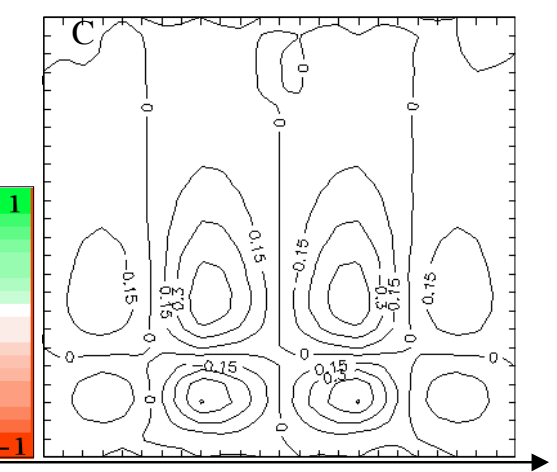

Figure 4. We can compare the measured $(A)$ and the modeled $(B, C)$ simple space-time separable cell's receptive fields. The model was made by the sum of two simple thalamic or cortical cells' response.

The different time course, dynamics of the simple cell's response can be obtained the same way as in the case of the lagged cells. The $\mathrm{x}$-t inseparable simple cells' transfer function can be modeled by two different ways. It can be constructed by the delayed convergence of two simple thalamic cells or the convergence of simple and lagged thalamic (cortical) cells. The previous method seems to have less biological reality then the later one, but in the model both of them works. The templates generating this type of receptive field are the followings:

$$
\begin{aligned}
& A_{57}=\left|\begin{array}{lll}
0 & 0 & 0 \\
0 & 1 & 0 \\
0 & 0 & 0
\end{array}\right| \quad A_{57 \text { (delayed 15) }}=\left|\begin{array}{ccc}
0 & 0 & 0 \\
0 & 0 & -1 \\
0 & 0 & 0
\end{array}\right| \\
& A_{57}=\left[\begin{array}{lll}
0 & 0 & 0 \\
0 & 1 & 0 \\
0 & 0 & 0
\end{array}\right] \quad A_{67 \text { (agged) }}=\left|\begin{array}{lll}
0 & 0 & 0 \\
0 & 0 & 1 \\
0 & 0 & 0
\end{array}\right|
\end{aligned}
$$

The modeled simple $\mathrm{x}$-t inseparable cells transfer function can be seen on Fig. 5 and Fig 6.

Time
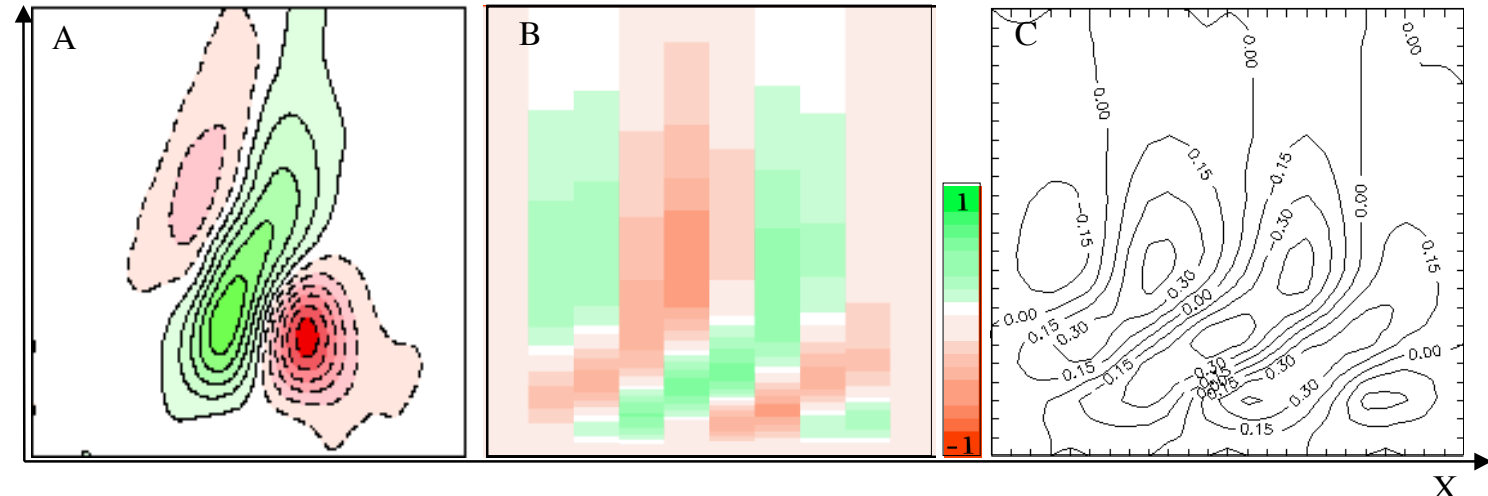

Figure 5. Compare the measured $(A)$ and the modeled $(B, C)$ space-time inseparable simple cell's receptive fields. In this case the model was made by the delayed $(d=15)$ sum of two simple cells' response.

The parameters of the templates are adjusted for the proper representation of the transfer function for a special magnitude of stimulus. The modeled transfer functions' characteristics can be provided by a wide range of parameter values as well. We could further decrease for example the number of the necessary layers (e.g., layer 1 and layer 4), but they were suitable for a better understanding of the underlying processes. 


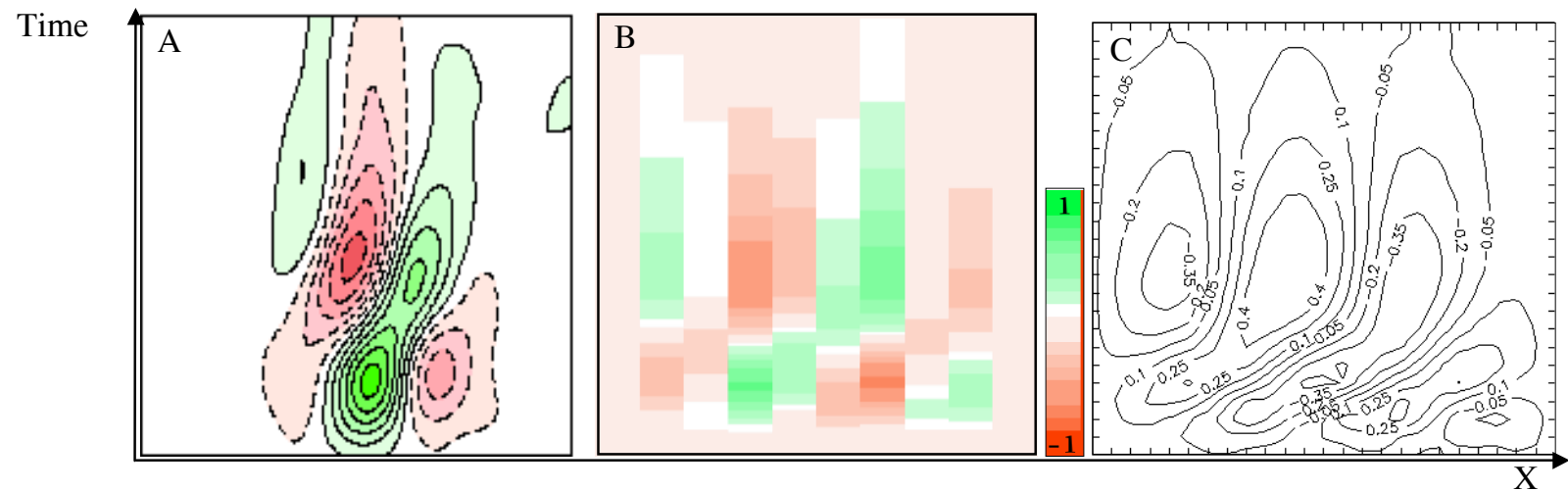

Figure 6. We can compare the measured $(A)$ and the modeled $(B B C) x$-t inseparable simple cell's receptive fields. In this case the model was made by the sum of a simple and a lagged cell's response.

\section{Summary}

Using relatively small (maximum neighborhood is 3) simple linear templates, we could construct a faithful CNN model of the transfer functions of the measured retinal ganglion cells, LGN neurons and of the simple cortical cells. Using networks of such model neurons it might be possible to reproduce the pattern of activity at different levels of visual information processing. This pattern of activity can help us in some practical CNN applications, such as compact coding, feature extraction or motion detection algorithms.

Modeling the dynamic receptive field structure of the central visual pathway neurons can improve our understanding of the visual information processing machinery. It can also partly uncover the unsolved mechanisms of the generation the RF's dynamic structure. The CNN models of the visual pathway neurons, can results in the construction of biology-inspired noise filtering methods, which reduce or appropriately redistribute transmission or compression noises.

\section{Acknowledgments}

The support of the grant No. T002578 (OTKA), and the comments and help of Marco Gilli are gratefully acknowledged.

\section{References}

[1] L. O. Chua, L. Yang: Cellular Neural Networks: Theory, IEEE Transactions on Circuits and Systems, Vol. 35, no. 10. 1988

[2] T. Roska, L. O. Chua: The CNN Universal Machine: An Analogic Array Computer, IEEE Transactions on Circuits and Systems-II: Analog and Digital Signal Processing, Vol. 40, no. 3. 1993

[3] G. C. De Angelis, I. Ohzawa, R.D. Freeman: Receptive-filed dymanics in the central visual pathways, Trends in Neurosciences (TINS), Vol. 18, no. 10, pp. 451-458. 1995

[4] T. Roska, J. Hámori, E. Lábos, K. Lotz, L. Orzó, J. Takács, P. L. Venetiáner, Z. Vidnyánszky, A. Zarándy: The Use of CNN Models in the subcortical Visual Pathway, IEEE Transactions on Circuits and Systems-I, Vol. 40, no. 3, pp. 182-193. 1993

[5] Hartline, H. K.: Am. J. Physiol. Vol. 130, pp. 690-699. 1940

[6] L.O. Chua and T. Roska: The CNN paradigm. IEEE Trans. CAS-I, Vol. 40, pp. 147-156. 1993

[7] T. Roska, J. Vandewalle (eds.): Cellular Neural Networks. J.Wiley and Sons, Chichester, London, New York 1993

[8] F. Werblin, A. Jacobs: Using CNN to unravel space-time processing in the vertebrate retina. CNNA-94, pp. 33-40. 1994

[9] [CNN-TOOLKIT] The cellular neural Network Workstation Toolkit, User's Manual, Version 6.0, Computer and Automation Institute, Hungarian Academy of Sciences, Budapest 1994

[10] W. Singer: Synchronization of cortical activity and its putative role in information processing and learning. Ann. Rev. Physiol.Vol. 55, pp. 349-74. 1993

[11] F. Werblin, T. Roska and L.O. Chua: "The Analogic Cellular Neural Network as a Bionic Eye". International journal of Circuit Theory and Applications, Vol. 23, pp. 541-569. 1995

[12] F. Werblin.: Synaptic connections, receptive fields and pattern of activityin the tiger salamander retina. Investigate opthalmology and visual science. Vol. 32, No. 3, pp.459-483. 1991 